$X=553=72-247$

PREPRINT

Miss $\mathrm{O}$ in 65964

POLAR MOTION FROM LASER TRACKING OF ARTIFICIAL SATELLITES

DAVID S SMITH RONALD K OLENKIEWICZ

PETER J DUNN

HENRY H PLOIKIN

THOMAS S JOHNSON

juLY 1972

NATIONAL TECHNICAL

INFORMATION SERVICE

GODDARD SPACE FLIGHT CENTER GREENBELT, MARYLAND

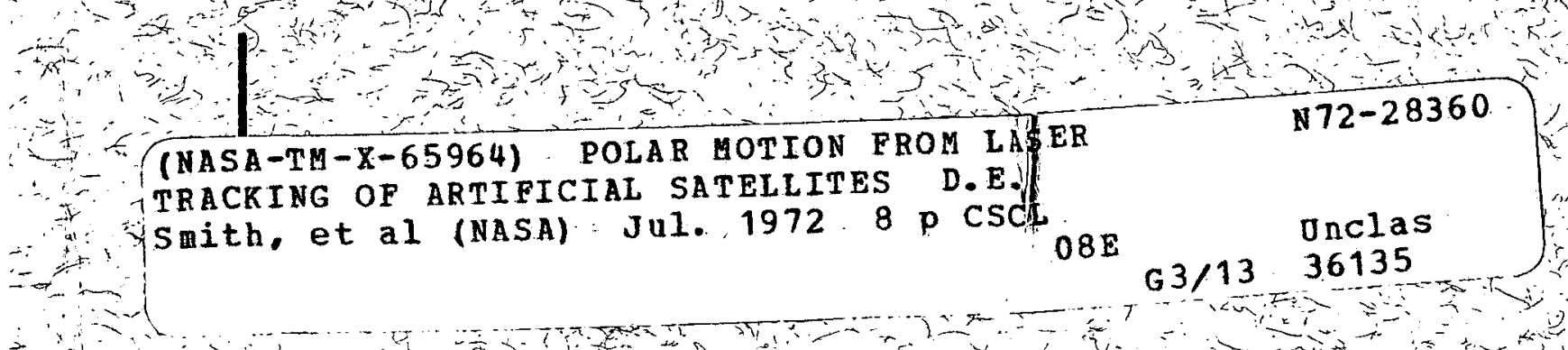


POLAR MOTION FROM LASER TRACKING OF ARTIFICIAL SATELLITES

David E. Smith and Ronald Kolenkiewicz

Trajectory Analysis and Geodynamics Division

Goddard Space Flight Center, Greenbelt, Maryland 20771

Peter J. Dunn

Wolf Research and Development Corporation 6801 Kenilworth Avenue, Riverdale, Maryland 20840

Henry H. Plotkin and Thomas S. Johnson

Advanced Data Systems Division

Goddard Space Flight Center, Greenbelt, Maryland 20771

July 1972

GODDARD SPACE FLIGHT CENTER

Greenbelt, Maryland 
PRECEDING PAGE BLANK NOT FLLMED

\title{
POLAR MOTION FROM LASER TRACKING OF ARTIFICIAL SATELLITES
}

\begin{abstract}
Laser ranges to the Beacon Explorer $\mathrm{C}$ spacecraft from a single Goddard Space Flight Center tracking system have been used to determine the change in latitude of the station arising from polar motion. A precision of 0.03 arcsecs $\mathrm{rms}$ was obtained for the
latitude during a five-month period in 1970 .
\end{abstract}




\section{POLAR MOTION FROM LASER TRACKING OF ARTIFICIAL SATELLITES}

A preliminary analysis of range data collected by a Goddard Space Flight Center laser tracking station during a 20-week period in 1970 has indicated that the orbital inclination of the Beacon Explorer C spacecraft can be determined to a precision of better than 0.03 seconds of arc from six hours of data. The significance of this result to the authors of this report is that it strongly suggests that a single laser tracking system can contribute measurements of changes in the position of the pole of rotation of the Earth in the meridian of the tracking station to a precision of at least 1 meter with a time resolution of $1 / 4$ of a day. The value of ultimately having high precision, high resolution polar motion information is, in part, in being able to fully investigate the correlation between earthquakes and polar motion (reference 1) and, partly, in a general improvement in our understanding and knowledge of the dynamics of the Earth. The present work is aimed towards achieving $10 \mathrm{~cm}$ polar position information with a time resolution of a few tenths of a day.

The laser tracking station that collected the data was situated at the GSFC Optical Facility in Greenbelt, Maryland. The laser is a 1 joule ruby system with a pulse rate of one-per-second and capable of ranging to specially equipped satellites carrying laser reflectors during both the day and the night (references 2,3 ). Tests of the laser ranging systems and comparisons of independent stations have shown that the measurement reproducibility and $\mathrm{rms}$ scatter is on the order of $\pm 30 \mathrm{~cm}$. A description of these tests is being prepared for publication. The satellite used in the experiment, Beacon Explorer C, has an orbit with inclination 41.1 degrees, an apogee of about $1300 \mathrm{~km}$ and a perigee of about $950 \mathrm{~km}$. The latitude of the tracking station is 39 degrees 01 minutes north and since this is similar to the orbital inclination of the BE-C satellite, the station was able to track the satellite on four consecutive passes each day (weather permitting), covering a time span of about six hours. During the other 18 hours, the spacecraft was generally below the horizon from the station.

A typical pass of the satellite over the station lasts about 10 minutes and on average about 300 ranges are obtained. During the tracking period that we have been investigating, July 6 to November 30,1970 , there were twenty-six occasions when four consecutive passes of the $\mathrm{BE}-\mathrm{C}$ satellite were obtained, yielding a total of nearly 20,000 range observations. Through each of these twenty-six four-pass configurations we fitted an orbit in which the Earth's gravitational field, complete through degree and order twelve, was modeled (reference 4), plus atmospheric drag (reference 5), solar radiation pressure, luni-solar gravitational perturbations, and the solid-earth tides. Because of the predominantly west to east motion of the satellite with respect to the tracking station the best determined parameter of the orbit was the inclination. Further, changes in the 
latitude of the tracking station would be reflected directly into the orbital inclination and, consequently, a detailed analysis of the changes in the inclination of $\mathrm{BE}-\mathrm{C}$ over a period of time might be expected to lead to the detection of polar motion in the meridian of the tracking station. This was the technique that was employed here and has been described in more detail in reference 6.

The values of the orbital inclination obtained from the four pass orbital arcs showed large systematic variations caused primarily by the gravitational effects of the sun, moon and solid-earth tides. After these had been subtracted and a resonance with the thirteenth order terms of the Earth's gravitational field removed, the resulting values of the orbital inclination showed an increase during the four-month period of approximately 0.37 arcseconds. These values are shown in Figure 1 as changes in the latitude of the Goddard laser. Also shown are the raw and smoothed changes in the latitude given by the Bureau International de l'Heure (BIH) (reference 7). The root-mean-square of the laser values about the smoothed $\mathrm{BIH}$ curve is $97 \mathrm{~cm}$, slightly less than 0.03 arcseconds, and the fit about a straight line through the laser data is $95 \mathrm{~cm}$. It is worth noting that the raw BIH positions are derived from observations made by up to 40 astronomical observatories using zenith telescopes.

In the analysis, the value of 0.25 was used for Love's tidal number $k_{2}$. This value was obtained by Anderle (reference 8) from Doppler tracking of several satellites and by Douglas et al (reference 9) from optical tracking of the GEOS satellites. The value is somewhat smaller than the 0.3 obtained from surface measurements (reference 10) but the lower figure appears to give a slightly better fit to our laser data. The importance of knowing the magnitude of $\mathrm{k}_{2}$ is evident when it is appreciated that the tidal perturbation of the orbital inclination of $\mathrm{BE}-\mathrm{C}$ is about 2 arcseconds full variation over a period of about three months. In the analysis, so far no attempt has been made to determine the best value of $k_{2}$ that fits the data and this is one area where further improvement in the results can be expected. Equally interesting has been the sensitivity of our results to the phase of the solid-earth tides. The initial calculations were all conducted with zero phase so that the peaks of the tidal bulges are directly beneath the sun and the moon. When a phase lag of 2.5 degrees was introduced into the tidal potential, significant improvement from about 0.05 arcseconds to 0.03 arcseconds ( 1.54 meters to 0.97 meters) was obtained in the fit of the laser values to the smoothed BIH curve. No other values of tidal lag have yet been tested but a careful inspection of the latitude residuals suggests that the original patterns that prompted the testing for phase have not yet been entirely removed. It is, of course, possible that the apparent phase lag is purely the result of not having used the correct value of $\mathrm{k}_{2}$ and for this reason, a re-investigation and determination of $\mathrm{k}_{2}$, and possibly the phase lag, is now underway. 
Also of considerable importance in the results is the basic quality of the laser data. There were twenty-six occasions when four consecutive passes were obtained during the time period being studied and there has been no indication in the data analysis that any of these values is the slightest way suspect. On the contrary, we feel it to be a significant achievement by the systems that the largest latitude residual is only 1.7 times the $\mathrm{rms}$, or about $160 \mathrm{~cm}$.

To summarize, laser data obtained in 1970 by a single GSFC tracking system has been used to monitor the changes in latitude of the station caused by polar motion. From data spans of six hours, the latitude of the station has been determined to a percision of better than one meter for a period of nearly five months. At the present time, this is probably the shortest averaging time over which the position of the pole has effectively been determined at the one meter level and gives some credence to the belief that high precision polar motion ( $10 \mathrm{~cm}$ averaged over one day) will be determinable from laser tracking in the near future.

\section{RE FERENCES}

1. D. E. Smylie and L. Mansinha, Journal of Geophysical Research, Vol. 73 No. 24, December 15, 1968.

2. T. S. Johnson, H. H. Plotkin, and P. L. Spadin, IEEE Journal of Quantum Electronics, Vol. QE-3, No. 11, November 1967.

3. S. T. Moss and H. H. Plotkin, "NASA Laser Systems Accuracy in Satellite Tracking, "Space Research X, Page 67, 1970, North Holland Publishing Company, Amsterdam.

4. D. E. Smith, F. J. Lerch, and C. A. Wagner, "A Gravitational Field Model for the Earth," Paper presented at Working Group 1, COSPAR, Madrid, May 1972.

5. L. G. Jacchia, Smithsonian Astrophysical Observatory, Special Report No. 332, May 1971.

6. D. E. Smith, R. Kolenkiewicz, and P. J. Dunn, "Geodetic Studies by Laser Ranging to Satellites," Paper presented at the Third International Symposium on Use of Artificial Satellites for Geodesy, Washington, D. C., April 1971.

7. B. Guinot, M. Feissel, and M. Granveaud, Annual Report for 1970, Bureau International de l'Heure, Paris, 1971. 
8. R. J. Anderle, NWL Technical Report No. TR-2889, August 1971.

9. B. C. Douglas, N. E. Mullins, and J. G. Marsh, "The Orbits of GEOS-I and -II, " Paper presented at Fall Annual Meeting of American Geophysical Union, December 1971.

10. P. Melchior, "Interaction Between Ocean and Earth Tides," Paper presented at Symposium on Marine Geodesy at the General Assembly IUGG, Moscow, August 1971. 


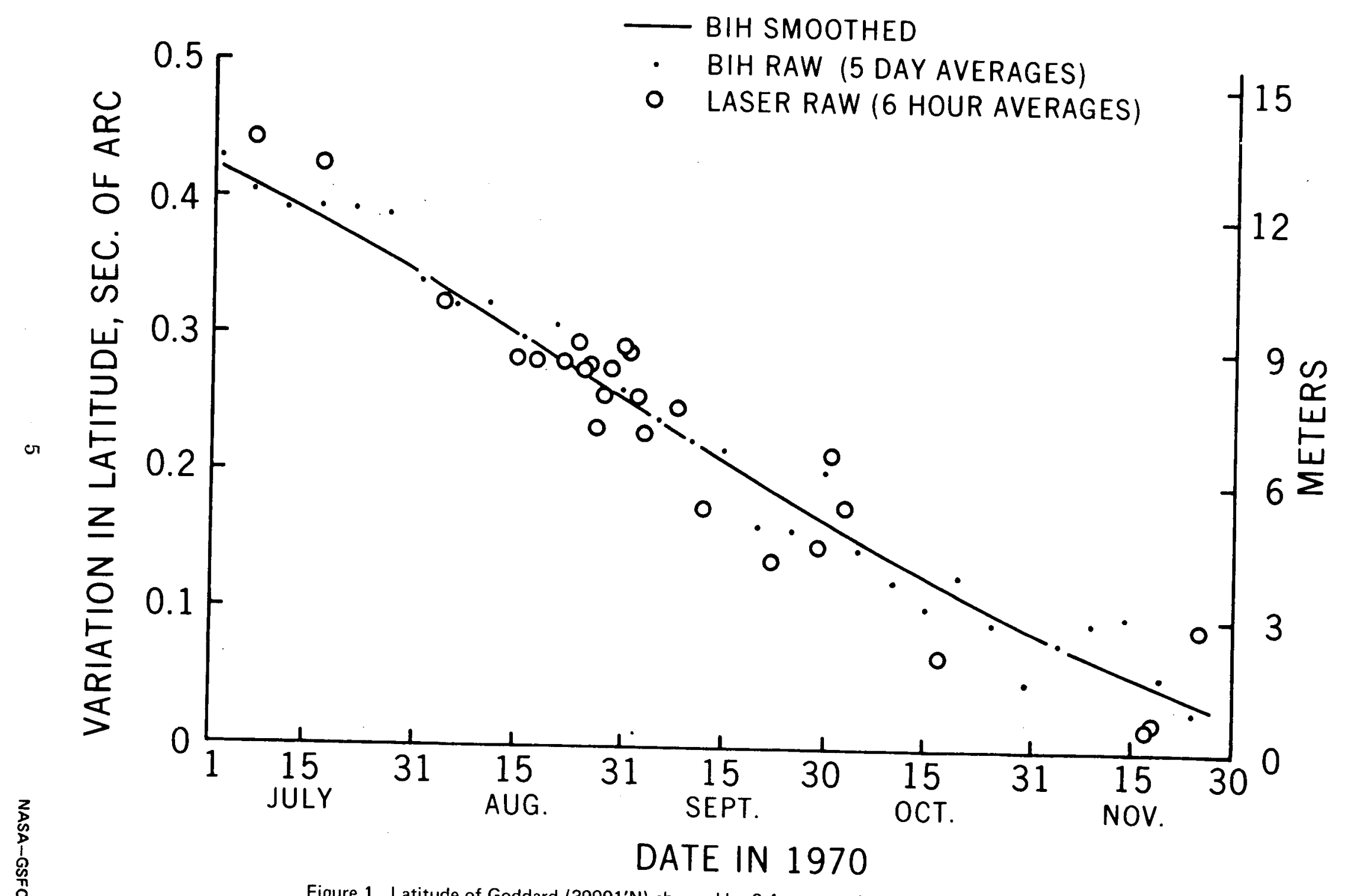

Figure 1. Latitude of Goddard (39001'N) changed by 0.4 arcesconds during the experiment.

RMS of laser residuals to the Bureau International de l'Heure $(\mathrm{BIH})$ smoothed curve is 0.03 arcseconds $(97 \mathrm{~cm})$. 